\title{
Known Galactic field Blazhko stars
}

\author{
M. Skarka \\ Department of Theoretical Physics and Astrophysics, Faculty of science, Masaryk University, Kotlářská 2, Brno, Czech Republic \\ e-mail: maska@physics.muni.cz
}

Received 18 September 2012 / Accepted 8 November 2012

\section{ABSTRACT}

\begin{abstract}
A list of known Galactic field stars exhibiting Blazhko effect containing 242 stars is presented. All the entries including their designations, positions, pulsation, and Blazhko periods were collected from the available literature. The actual values of parameters are given.
\end{abstract}

Key words. catalogs - stars: horizontal-branch - stars: variables: RR Lyrae

\section{Introduction}

In the era of automatic sky surveys and space telescopes, it is increasingly obvious that there is a high percentage of RR Lyrae type stars exhibiting the Blazhko effect (more than $40 \%$, Kolenberg et al. 2010). The light curves of such stars are amplitude- or phase-modulated with the periods typically in the order of tens to hundreds of days ${ }^{1}$. This behaviour is named after one of its discoverers, S. N. Blazhko (1907), who noticed it in RW Dra.

Many theories, such as resonances between radial and nonradial modes (Dziembowski \& Mizerski 2004), effects connected with convection (Stothers 2006), etc., have tried to describe observed properties, but the explanation of the nature of the Blazhko effect is still missing. A brief overview of the Blazhko effect can be found in Kovács (2009) and Kolenberg (2012). Nowadays, the most likely explanation of the Blazhko effect is considered resonance between the fundamental radial mode and the ninth overtone (Buchler \& Kolláth 2011).

Some partial lists of the Blazhko stars have been published (e.g. Smith 1995; Sódor \& Wils 2005; Le Borgne et al. 2012), but an overall list has not been available until now. Such a list allows observers and all interested astronomers to quickly check the Blazhko instability of the star in one place and see whether the star needs further observations or not.

\section{The list}

The list contains values based on the data from sky surveys ASAS (Szczygiel \& Fabrycky 2007) and NSVS (Wils et al. 2006), as well as the data based on O-C diagram analyses (Le Borgne et al. 2012), and finally it contains values obtained in detailed studies of many of these stars. Stars with only one modulation period are listed in Table 1, stars with multiple Blazhko period are in Table 2, and RR Lyraes with variable Blazhko period are in Table 3.

Coordinates and magnitude ranges were taken from the VSX database (Watson et al. 2006). If there is more than one available value of the Blazhko period, then the value with the highest priority is given. The hierarchy of cited references is the following: Values taken from detail studies have the highest priority, data from surveys have lower priority, and the the data based on $\mathrm{O}-\mathrm{C}$ studies the lowest priority. There are some exceptions, mainly if the data with higher priority were published before 1990 or if the data are of worse quality than values with lower priority. If the values of Blazhko periods differ more than one day, then all available values are listed.

The stars are sorted by right ascension. If possible, the ASAS and other designations of the stars were transformed to GCVS names. This is the case of V1820 Ori, BB Lep, V339 Lup, MR Lib, V559 Hya, V552 Hya, V701 Pup, LR Eri, IY Eri, GW Cet, DZ Oct, V354 Vir, V419 Vir, V476 Vir, V551 Vir, OR Com, BT Sco, V1319 Sco, BT Ant, AD UMa, NS UMa, PP UMa, KV Cnc, AI Crt, and FR Psc, which were noted in Wils et al. (2006) and Szczygiel \& Fabrycky (2007) in other forms.

There are three stars with special characteristics in the list. These objecst deserve to be observed as a matter of priority. Some indications show that BV Aqr is a RRd type (Jerzykiewicz 1995). VX Her is suspected to be a member of an eclipsing binary star (Fitch et al. 1966), which is the first possible occurrence of such objects among RR Lyraes. SU Col probably has three modulation periods, which is also unique behaviour (Szczygiel $\&$ Fabrycky 2007). Some stars have a very short period (less than $0.23 \mathrm{~d}$ ) or amplitude (about 0.1 ). These objects should be observed first.

Objects with very long (more than $1000 \mathrm{~d}$ ) Blazko period are marked by a colon in the second column. The same mark may be used for stars that are only suspected of the Blazhko effect or whose Blazhko period is not well determined. A regularly updated list with Tables $1-3$ is also available on the web page http://physics .muni.cz/ blasgalf.

Acknowledgements. Work on the paper have been supported by GACR project GD205/08/H005, MU MUNI/A/0968/2009. The International Variable Star Index (VSX) database, operated at AAVSO, Cambridge, Massachusetts, USA have been used. This research also have made use of VizieR catalogue access tool, CDS, Strasbourg, France. I would like to thank Miloslav Zejda for usefull comments and suggestions.

1 There are also several stars with changing Blazhko effect. 
Table 1. List of Blazhko stars with one modulation period.

\begin{tabular}{|c|c|c|c|c|c|c|c|c|c|}
\hline Star & RA & Dec & Type & $\begin{array}{c}V_{\max } \\
{[\mathrm{mag}]}\end{array}$ & $\begin{array}{c}V_{\min } \\
{[\mathrm{mag}]}\end{array}$ & $\begin{array}{c}P_{\text {puls }} \\
\text { [d] }\end{array}$ & Ref. & $\begin{array}{l}P_{\mathrm{Bl}} \\
{[\mathrm{d}]}\end{array}$ & Ref. \\
\hline RY Psc & 001141.10 & -014455.3 & RRab & 11.82 & 12.72 & 0.5297456 & 25 & 154.53 & 25 \\
\hline OV And: & 002044.86 & 404941.8 & $\mathrm{RRab}$ & 10.90 & 11.26 & 0.47060 & 3 & 27 & 27 \\
\hline SW And: & 002343.09 & 292403.6 & $\mathrm{RRab}$ & 9.14 & 10.09 & 0.4422618 & 3 & 36.8 & 26 \\
\hline RX Cet & 003338.28 & -152914.9 & RRab & 11.01 & 11.75 & 0.5736918 & 18 & 255.5 & 25 \\
\hline ASAS003514-0415.0: & 003514.00 & -041500.0 & $\mathrm{RRc}$ & 12.92 & 13.73 & 0.3445751 & 25 & 1616.29 & 25 \\
\hline ASAS003706-4317.7 & 003706.00 & -431742.0 & RRab & 13.29 & 14.60 & 0.6275343 & 25 & 187.12 & 25 \\
\hline SW Psc: & 004119.41 & 052047.0 & $\mathrm{RRab}$ & 13.3 & 14.9 & 0.521265 & 3 & 34.5 & 26 \\
\hline FR Psc: & 004757.06 & 114243.5 & $\mathrm{RRab}$ & 11.5 & 12.8 & 0.45568 & 3 & 55 & 27 \\
\hline RU Cet: & 010040.30 & -155727.6 & $\mathrm{RRab}$ & 11.10 & 12.03 & 0.5862844 & 3 & 98 & 26 \\
\hline ET Cep & 010223.30 & 852349.2 & RRab & 13.5 & 14.5 & 0.49716 & 3 & $\ldots$ & 37 \\
\hline DR And: & 010510.71 & 341306.3 & $\mathrm{RRab}$ & 12.03 & 13.15 & 0.563118 & 15 & $\sim 57.5$ & 15 \\
\hline CS Phe & 010949.45 & -441853.5 & RRab & 12.66 & 13.53 & 0.4843964 & 3 & 62.5 & 17 \\
\hline RU Psc: & 011426.04 & 242456.4 & $\mathrm{RRc}$ & 9.93 & 10.40 & 0.390385 & 3 & 28 & 26 \\
\hline AM Tuc: & 011830.65 & -675505.0 & $\mathrm{RRc}$ & 11.39 & 11.87 & 0.4057948 & 25 & 1748.86 & 25 \\
\hline$X Y$ And & 012642.41 & 340407.4 & RRab & 12.90 & 14.22 & 0.3987247 & 9 & 41.37 & 9 \\
\hline GW Cet & 012848.27 & -112712.6 & RRab & 12.4 & 13.9 & 0.516648 & 3 & 84.99 & 25 \\
\hline ASAS013140-4957.3 & 013140.60 & -495718.9 & RRab & 12.12 & 13.22 & 0.464329 & 25 & 40.17 & 25 \\
\hline UX Tri & 014535.01 & 312249.6 & RRab & 13.07 & 14.50 & 0.4669218 & 13 & 43.7 & 13 \\
\hline IY Eri: & 020728.18 & -575209.5 & $\mathrm{RRc}$ & 10.86 & 11.31 & 0.3750261 & 25 & 1673.36 & 25 \\
\hline SS For & 020751.98 & -265157.7 & RRab & 9.45 & 10.60 & 0.495433 & 19 & 34.94 & 19 \\
\hline RV Cet & 021514.90 & -104800.7 & RRab & 10.35 & 11.22 & 0.6234139 & 25 & 112.05 & 25 \\
\hline ASAS022637-4119.7 & 022637.00 & $-41 \quad 1942.0$ & $\mathrm{RRc}$ & 10.08 & 10.21 & 0.2941932 & 25 & 357.94 & 25 \\
\hline RV Hor & 025020.47 & -641540.9 & $\mathrm{RRab}$ & 12.9 & 14.5 & 0.5724975 & 25 & 79.81 & 25 \\
\hline ASAS030534-3116.1 & 030534.00 & -311606.0 & $\mathrm{RRab}$ & 12.53 & 14.00 & 0.4964538 & 25 & 6.77 & 25 \\
\hline RX For & 031113.22 & -262858.8 & $\mathrm{RRab}$ & 11.12 & 12.46 & 0.59731 & 18 & 31.79 & 23 \\
\hline ASAS031408-3446.4: & 031408.00 & -344624.0 & RRc & 11.54 & 12.07 & 0.3124235 & 25 & 1241.77 & 25 \\
\hline ASAS032438-2334.7 & 032438.00 & -233442.0 & RRab & 12.09 & 13.03 & 0.6296339 & 25 & 335.29 & 25 \\
\hline X Ret & 032520.10 & -650318.6 & RRab & 11.16 & 12.14 & 0.4920082 & 25 & 160.64 & 25 \\
\hline ASAS033108+0713.4: & 033108.38 & 071324.9 & $\mathrm{RRab}$ & 10.74 & 10.87 & 0.5281963 & 25 & 1442.79 & 25 \\
\hline LR Eri & 040010.76 & -194937.1 & $\mathrm{RRab}$ & 12.0 & 13.0 & 0.60225 & 17 & 122 & 17 \\
\hline FM Per: & 040327.06 & 475951.7 & $\mathrm{RRab}$ & 12.02 & 13.24 & 0.489256 & 14 & $\sim 122$ & 14 \\
\hline & & & & & & & & 20 & 27 \\
\hline AH Cam & 040638.89 & 552959.7 & RRab & 11.31 & 12.33 & 0.3687346 & 3 & 10.83 & 23 \\
\hline XY Eri & 041116.78 & -135054.3 & $\mathrm{RRab}$ & 12.39 & 13.27 & 0.55426 & 18 & 50.2 & 23 \\
\hline BR Tau & 043442.91 & 214621.7 & RRab & 12.07 & 13.38 & 0.3905928 & 8 & 19.3 & 8 \\
\hline AL Pic & 044130.80 & -521637.0 & RRab & 12.8 & 14.0 & 0.54861 & 18 & 34 & 17 \\
\hline $\mathrm{U} \mathrm{Cae}$ & 045314.41 & -374915.9 & $\mathrm{RRab}$ & 11.43 & 12.70 & 0.4197835 & 3 & 22.8 & 23 \\
\hline NSV1856 & 050838.65 & -560257.5 & RRab & 12.5 & 13.5 & 0.5160761 & 25 & 786.91 & 25 \\
\hline RY Col & 051507.78 & -413741.7 & RRab & 10.44 & 11.24 & 0.4788368 & 25 & 82.08 & 23 \\
\hline ASAS052402-2247.4 & 052402.00 & -224724.0 & RRab & 13.40 & 14.59 & 0.6498517 & 25 & 10.23 & 25 \\
\hline ASAS052840-5316.2 & 052840.00 & -531612.0 & $\mathrm{RRc}$ & 13.38 & 14.05 & 0.3678062 & 25 & 453.43 & 25 \\
\hline ASAS053022-3234.8 & 053022.00 & -323448.0 & $\mathrm{RRc}$ & 11.77 & 11.97 & 0.2331114 & 25 & 364.6 & 25 \\
\hline ASAS053628-3837.0: & 053628.00 & -383700.0 & $\mathrm{RRc}$ & 12.78 & 13.23 & 0.3714727 & 25 & 1394.31 & 25 \\
\hline BB Lep & 054230.18 & -162254.5 & RRab & 11.85 & 12.71 & 0.5389135 & 25 & 22.84 & 25 \\
\hline ASAS054843-1627.0: & 054843.00 & -162700.0 & $\mathrm{RRab}$ & 12.96 & 13.57 & 0.3767273 & 25 & 1663.06 & 25 \\
\hline ASAS055322-5417.9 & 055322.00 & -541754.0 & $\mathrm{RRc}$ & 12.82 & 13.38 & 0.2452638 & 25 & 381.58 & 25 \\
\hline V1820 Ori & 055437.13 & 045411.4 & $\mathrm{RRab}$ & 12.5 & 13.4 & 0.47927 & 3 & 28 & 27 \\
\hline VW Dor & 060745.71 & -665838.8 & $\mathrm{RRab}$ & 11.1 & 12.25 & 0.57057 & 18 & 25.99 & 23 \\
\hline \multirow[t]{2}{*}{$\mathrm{RX} \mathrm{Col}$} & 061314.74 & -371500.6 & $\mathrm{RRab}$ & 12.32 & 12.95 & 0.59376 & 18 & 130 & 17 \\
\hline & & & & & & & & 137.77 & 23 \\
\hline
\end{tabular}


Table 1. continued.

\begin{tabular}{|c|c|c|c|c|c|c|c|c|c|}
\hline Star & RA & Dec & Type & $\begin{array}{c}V_{\max } \\
{[\mathrm{mag}]}\end{array}$ & $\begin{array}{c}V_{\min } \\
{[\mathrm{mag}]}\end{array}$ & $\begin{array}{c}P_{\text {puls }} \\
\text { [d] }\end{array}$ & Ref. & $\begin{array}{l}P_{\mathrm{Bl}} \\
{[\mathrm{d}]}\end{array}$ & Ref. \\
\hline ST Pic & 061401.17 & -612823.5 & RRab & 9.29 & 9.77 & 0.4857445 & 25 & 117.9 & 25 \\
\hline ASAS062326+0005.8 & 062326.00 & 000548.0 & RRab & 11.96 & 12.59 & 0.551317 & 25 & 37.24 & 25 \\
\hline ASAS064615-4319.2: & 064615.00 & -431912.0 & $\mathrm{RRc}$ & 12.93 & 13.63 & 0.3186021 & 25 & 1639.61 & 25 \\
\hline ASAS070001-3732.5 & 070000.65 & -373231.5 & $\mathrm{RRab}$ & 11.85 & 12.74 & 0.4941843 & 25 & 116.96 & 25 \\
\hline ASAS070854+1919.7 & 070853.85 & 191938.0 & $\mathrm{RRab}$ & 8.69 & 8.79 & 0.7789192 & 25 & 7.92 & 25 \\
\hline ASAS071549-4405.3 & 071549.00 & -440518.0 & $\mathrm{RRc}$ & 13.04 & 13.67 & 0.3144609 & 25 & 488.4 & 25 \\
\hline RR Gem & 072133.53 & 305259.5 & $\mathrm{RRab}$ & 10.62 & 11.99 & 0.3972884 & 4 & 7.23 & 4 \\
\hline ASAS080249-5913.5: & 080248.94 & -591328.0 & $\mathrm{RRc}$ & 11.75 & 12.00 & 0.3541891 & 25 & 1185.26 & 25 \\
\hline ASAS080318-2530.1: & 080318.25 & -253006.3 & RRc & 12.5 & 13.0 & 0.2697723 & 25 & 1542.02 & 25 \\
\hline SS Cnc & 080625.59 & 231505.7 & $\mathrm{RRab}$ & 11.49 & 12.72 & 0.367337 & 2 & 5.309 & 2 \\
\hline DD Hya & 081231.81 & 025005.0 & $\mathrm{RRab}$ & 11.57 & 12.71 & 0.501776 & 3 & 34.59 & 23 \\
\hline V701 Pup & 081932.86 & -235809.8 & $\mathrm{RRc}$ & 10.45 & 10.75 & 0.2856671 & 25 & 8.1 & 25 \\
\hline NS UMa: & 082424.73 & 654303.4 & $\mathrm{RRab}$ & 10.75 & 11.35 & 0.59910 & 3 & 65 & 27 \\
\hline TT Cnc & 083255.18 & 131128.5 & $\mathrm{RRab}$ & 10.93 & 11.57 & 0.56340 & 23 & 89.02 & 23 \\
\hline KV Cnc: & 084002.42 & 274331.6 & $\mathrm{RRab}$ & 11.9 & 13.0 & 0.50200 & 3 & 42 & 27 \\
\hline SV Vol & 084832.64 & -713914.8 & RRab & 11.78 & 12.57 & 0.6099118 & 3 & 85.47 & 23 \\
\hline PP UMa: & 085215.07 & 702623.9 & RRab & 13.45 & 14.35 & 0.51869 & 3 & 46 & 27 \\
\hline ASAS085254-0300.3 & 085254.00 & -030018.0 & $\mathrm{RRc}$ & 12.42 & 12.65 & 0.2669022 & 25 & 11.8 & 25 \\
\hline DZ Oct & 085448.33 & -831657.2 & $\mathrm{RRab}$ & 12.2 & 13.5 & 0.47786 & 18 & 36.8 & 25 \\
\hline ASAS090900-0410.4 & 090900.10 & -041024.0 & $\mathrm{RRc}$ & 10.68 & 11.09 & 0.3032613 & 25 & 8.52 & 25 \\
\hline SZ Hya & 091348.68 & -09 1908.9 & $\mathrm{RRab}$ & 10.44 & 11.84 & 0.53724022 & 3 & 26.23 & 23 \\
\hline RW Cnc: & 091906.04 & 290355.7 & $\mathrm{RRab}$ & 10.7 & 12.6 & 0.547199 & 3 & 87 & 26 \\
\hline UU Hya & 093629.73 & 040640.3 & $\mathrm{RRab}$ & 11.73 & 12.73 & 0.5238684 & 3 & 39.89 & 23 \\
\hline ASAS093731-1816.2 & 093731.00 & -181612.0 & $\mathrm{RRab}$ & 13.05 & 14.28 & 0.529175 & 25 & 87.73 & 25 \\
\hline CM UMa & 094313.78 & 492937.3 & $\mathrm{RRab}$ & 12.8 & 13.8 & 0.589124 & 10 & 27.77 & 10 \\
\hline CD Vel & 094438.24 & -455237.2 & RRab & 11.3 & 12.4 & 0.5735076 & 25 & 66.35 & 25 \\
\hline ASAS101200+1921.9: & 101200.00 & 192154.0 & $\mathrm{RRab}$ & 11.55 & 12.43 & 0.4826394 & 25 & 1141.03 & 25 \\
\hline Y LMi: & 101551.45 & 325132.5 & $\mathrm{RRab}$ & 11.4 & 13.3 & 0.524471 & 3 & 33.4 & 26 \\
\hline Cze V134: & 102226.27 & 591236.2 & $\mathrm{RRc}$ & 11.46 & 11.63 & 0.419794 & 3 & $\ldots$ & 44 \\
\hline V543 Нyа & 102608.40 & -231513.9 & $\mathrm{RRab}$ & 12.8 & 13.9 & 0.59826 & 17 & 59 & 17 \\
\hline BT Ant: & 103202.59 & -301037.3 & $\mathrm{RRc}$ & 11.55 & 12.02 & 0.3304459 & 25 & 1731.6 & 25 \\
\hline AF Vel & 105302.49 & -495422.7 & $\mathrm{RRab}$ & 10.68 & 11.78 & 0.5274139 & 25 & 58.55 & 23 \\
\hline SZ Leo & 110136.82 & 080955.6 & $\mathrm{RRab}$ & 11.79 & 12.72 & 0.53408 & 17 & 179 & 17 \\
\hline AH Leo: & 110505.29 & 232108.4 & $\mathrm{RRab}$ & 13.67 & 14.66 & 0.4662609 & 1 & $\sim 20$ & 1 \\
\hline ASAS110522-2641.0 & 110522.00 & -264100.0 & $\mathrm{RRc}$ & 11.68 & 12.06 & 0.2944559 & 25 & 7.4 & 25 \\
\hline ASAS112027-4338.8: & 112026.70 & -433848.0 & $\mathrm{RRc}$ & 11.05 & 11.34 & 0.3795948 & 25 & 1480.38 & 25 \\
\hline AI Crt: & 112607.49 & -140343.1 & $\mathrm{RRab}$ & 15.5 & 16.1 & 0.50290 & 17 & 63 & 17 \\
\hline V354 Vir: & 114332.22 & 024155.6 & $\mathrm{RRab}$ & 12.4 & 13.3 & 0.59503 & 3 & 59 & 27 \\
\hline BI Cen & 114554.65 & -592240.2 & $\mathrm{RRab}$ & 11.18 & 12.33 & 0.4531949 & 3 & 79.68 & 23 \\
\hline $\mathrm{X} \mathrm{Crt}$ & 114856.22 & -102628.6 & $\mathrm{RRab}$ & 11.09 & 11.75 & 0.73284 & 17 & 143 & 17 \\
\hline \multirow[t]{2}{*}{ IK Hya: } & 120447.27 & -274043.3 & $\mathrm{RRab}$ & 9.96 & 10.42 & 0.653243 & 25 & 72 & 17 \\
\hline & & & & & & & & 67.5 & 23 \\
\hline EL Hya: & 120942.07 & -345726.3 & $\mathrm{RRc}$ & 13.3 & 13.8 & 0.3436271 & 25 & 1611.6 & 25 \\
\hline V552 Нуа & 121206.12 & -261248.2 & $\mathrm{RRab}$ & 12.5 & 13.8 & 0.39878 & 17 & 48.3 & 17 \\
\hline TU Com: & 121346.92 & 305907.5 & $\mathrm{RRab}$ & 12.9 & 16.4 & 0.461809 & 3 & $\sim 75.00$ & 3 \\
\hline SV Hya & 123030.50 & -260251.1 & RRab & 9.78 & 11.00 & 0.4785475 & 25 & 63.29 & 25 \\
\hline ASAS123812-4422.5: & 123812.50 & -442230.0 & $\mathrm{RRab}$ & 13.1 & 13.82 & 0.523549 & 25 & 1307.7 & 25 \\
\hline V419 Vir & 124804.51 & -082047.4 & RRab & 11.98 & 12.86 & 0.515287 & 25 & 65.69 & 25 \\
\hline $\mathrm{ZCVn}$ & 124945.42 & 434625.6 & $\mathrm{RRab}$ & 11.43 & 12.36 & 0.653819 & 3 & 22.98 & 23 \\
\hline AS Vir: & 125245.86 & -101536.4 & $\mathrm{RRab}$ & 11.60 & 12.23 & 0.55340399 & 3 & $\ldots$ & 34 \\
\hline
\end{tabular}


Table 1. continued.

\begin{tabular}{|c|c|c|c|c|c|c|c|c|c|}
\hline Star & RA & Dec & Type & $\begin{array}{c}V_{\max } \\
{[\mathrm{mag}]}\end{array}$ & $\begin{array}{c}V_{\min } \\
{[\mathrm{mag}]}\end{array}$ & $\begin{array}{c}P_{\text {puls }} \\
\text { [d] }\end{array}$ & Ref. & $\begin{array}{l}P_{\mathrm{Bl}} \\
{[\mathrm{d}]}\end{array}$ & Ref \\
\hline RY Com & 130507.99 & 231642.2 & RRab & 11.75 & 12.92 & 0.468951 & 8 & 32 & 8 \\
\hline UZ Vir & 130844.34 & 132408.3 & $\mathrm{RRab}$ & 12.58 & 13.70 & 0.4593925 & 9 & 68.24 & 9 \\
\hline OR Com & 131954.50 & 195336.8 & RRab & 12.6 & 13.7 & 0.601167 & 3 & 74 & 27 \\
\hline AM Vir: & 132333.33 & -163957.9 & RRab & 11.16 & 11.85 & 0.61509 & 17 & 49.8 & 17 \\
\hline V476 Vir: & 132922.48 & -055259.2 & $\mathrm{RRab}$ & 11.2 & 12.1 & 0.5763497 & 25 & 1398.99 & 25 \\
\hline SS CVn & 134815.94 & 395403.0 & $\mathrm{RRab}$ & 11.53 & 12.62 & 0.47851 & 3 & 93.72 & 23 \\
\hline ASAS135740-1202.3: & 135740.00 & -120218.0 & RRc & 12.30 & 12.71 & 0.2671226 & 25 & 1189.91 & 25 \\
\hline ASAS135813-4215.1 & 135813.00 & -421506.0 & $\mathrm{RRab}$ & 12.43 & 13.27 & 0.5231816 & 25 & 146.01 & 25 \\
\hline V674 Cen: & 140324.08 & -362420.1 & $\mathrm{RRab}$ & 11.0 & 11.9 & 0.4939666 & 25 & 1650.71 & 25 \\
\hline ASAS141025-2244.8: & 141025.00 & -224448.0 & $\mathrm{RRab}$ & 12.60 & 13.29 & 0.6398808 & 25 & 1556.66 & 25 \\
\hline V559 Нyа & 141345.50 & -225441.9 & $\mathrm{RRab}$ & 11.9 & 13.1 & 0.44794 & 17 & 26.6 & 17 \\
\hline TV Boo & 141636.58 & 422135.7 & RRc & 10.71 & 11.30 & 0.31255936 & 3 & 10 & 27 \\
\hline V551 Vir & 142305.58 & 015400.9 & $\mathrm{RRab}$ & 12.7 & 13.8 & 0.44692 & 3 & 48 & 27 \\
\hline SW Boo: & 142734.86 & 360244.1 & $\mathrm{RRab}$ & 11.76 & 12.88 & 0.5135281 & 3 & 13 & 26 \\
\hline ST Vir & 142739.08 & 005405.8 & $\mathrm{RRab}$ & 10.84 & 12.15 & 0.4108143 & 3 & 25.58 & 23 \\
\hline RS Boo & 143333.21 & $314516.6 a ̆$ & $\mathrm{RRab}$ & 9.69 & 10.84 & 0.37733896 & 3 & 532.48 & 23 \\
\hline ASAS144154-0324.7 & 144154.00 & -032442.0 & RRc & 11.40 & 11.72 & 0.2293674 & 25 & 5.65 & 25 \\
\hline TY Aps & 144850.01 & -711941.9 & $\mathrm{RRab}$ & 11.20 & 12.43 & 0.5016935 & 3 & 109.13 & 23 \\
\hline MR Lib & 145315.44 & -143556.8 & $\mathrm{RRab}$ & 12.4 & 13.4 & 0.54007 & 17 & 41.7 & 17 \\
\hline V339 Lup & 150327.43 & -475603.7 & RRab & 11.5 & 12.3 & 0.60058 & 17 & 59.5 & 17 \\
\hline FU Lup & 150923.77 & -431937.1 & $\mathrm{RRab}$ & 14.0 & 15.0 & 0.3821508 & 25 & 42.49 & 25 \\
\hline ASAS151849-1000.0 & 151849.00 & -100000.0 & $\mathrm{RRc}$ & 12.03 & 12.53 & 0.3364272 & 25 & 802.95 & 25 \\
\hline ST Boo & 153039.23 & 354704.3 & $\mathrm{RRab}$ & 10.49 & 11.41 & 0.62229069 & 3 & 284.09 & 23 \\
\hline AR Ser: & 153330.82 & 024637.9 & $\mathrm{RRab}$ & 11.6 & 12.2 & 0.5752124 & 3 & 63 & 27 \\
\hline CG Lib: & 153516.81 & -242012.5 & $\mathrm{RRc}$ & 11.2 & 11.8 & 0.306777 & 25 & 1560.06 & 25 \\
\hline ASAS153830-6906.4 & 153830.00 & -690624.0 & $\mathrm{RRab}$ & 12.26 & 13.15 & 0.6224747 & 25 & 118.05 & 25 \\
\hline V1141 Her: & 155458.55 & 424610.5 & RRc & 10.97 & 11.56 & 0.317152 & 3 & $\sim 30$ & 45 \\
\hline V1319 Sco: & 155551.59 & -214832.9 & $\mathrm{RRc}$ & 11.35 & 11.90 & 0.2541338 & 25 & 1699.52 & 25 \\
\hline PQ Lup & 155553.25 & -404143.6 & $\mathrm{RRab}$ & 11.6 & 12.4 & 0.58198 & 17 & 48.8 & 17 \\
\hline AR Her & 160032.23 & 465525.7 & $\mathrm{RRab}$ & 10.59 & 11.63 & 0.470028 & 3 & 32 & 27 \\
\hline BT Sco & 161255.58 & -082728.0 & $\mathrm{RRab}$ & 12.61 & 13.40 & 0.54871 & 17 & 78 & 17 \\
\hline GSC02050-00745 & 161834.34 & 272813.2 & $\mathrm{RRab}$ & 14.27 & $\ldots$ & 0.508646 & 3 & $\ldots$ & 36 \\
\hline BS Aps & 162051.50 & -714015.8 & RRab & 11.85 & 12.49 & 0.5825589 & 3 & 40.93 & 23 \\
\hline ASAS162158+0244.5 & 162158.00 & 024430.0 & RRc & 12.47 & 12.99 & 0.3238044 & 25 & 8.11 & 25 \\
\hline ASAS162811+0304.3 & 162811.00 & 030418.0 & $\mathrm{RRab}$ & 13.09 & 14.74 & 0.5970104 & 25 & 26.28 & 25 \\
\hline VX Her & 163040.80 & 182200.6 & $\mathrm{RRab}$ & 9.91 & 11.18 & 0.4553573 & 3 & 455.37 & 39 \\
\hline \multirow[t]{3}{*}{ UV Oct: } & 163225.53 & -835410.5 & $\mathrm{RRab}$ & 8.70 & 9.97 & 0.542625 & 3 & 143.73 & 25 \\
\hline & & & & & & & & 145 & 17 \\
\hline & & & & & & & & 146.99 & 23 \\
\hline RW Dra & 163531.60 & 575023.2 & RRab & 11.05 & 12.08 & 0.442917 & 3 & 41.42 & 23 \\
\hline ASAS170223-2422.0 & 170223.18 & -242159.2 & $\mathrm{RRab}$ & 11.34 & 11.73 & 0.4613693 & 25 & 22.18 & 25 \\
\hline V1124 Her & 170432.90 & 142633.0 & $\mathrm{RRab}$ & 12.10 & 12.95 & 0.55102 & 27 & 39 & 27 \\
\hline V365 Her: & 170539.86 & 213058.0 & $\mathrm{RRab}$ & 12.61 & 13.55 & 0.3797141 & 3 & 40 & 27 \\
\hline DL Her & 172022.45 & 143038.7 & $\mathrm{RRab}$ & 11.72 & 12.63 & 0.5916369 & 3 & 34 & 27 \\
\hline ASAS172721-5305.9 & 172721.00 & -530554.0 & $\mathrm{RRab}$ & 12.30 & 13.55 & 0.435433 & 25 & 58.66 & 25 \\
\hline EZ Ara: & 172931.78 & -554818.7 & RRc & 12.9 & 13.7 & 0.3273052 & 25 & 1610.05 & 25 \\
\hline V421 Her: & 173205.47 & 394532.2 & $\mathrm{RRab}$ & 13.33 & 14.46 & 0.55677 & 3 & 56 & 27 \\
\hline V788 Oph: & 173609.07 & 080954.1 & $\mathrm{RRab}$ & 13.3 & 14.9 & 0.547131 & 3 & 115 & 3 \\
\hline V434 Her: & 174033.01 & 224902.3 & $\mathrm{RRab}$ & 13.8 & 15.2 & 0.5144034 & 3 & 26.1 & 26 \\
\hline V494 Sco: & 174048.48 & -313231.8 & $\mathrm{RRab}$ & 10.62 & 11.91 & 0.427297 & 25 & 455 & 17 \\
\hline
\end{tabular}


Table 1. continued.

\begin{tabular}{|c|c|c|c|c|c|c|c|c|c|}
\hline$\overline{\text { Star }}$ & $\overline{\mathrm{RA}}$ & Dec & Type & $\begin{array}{c}V_{\max } \\
{[\mathrm{mag}]}\end{array}$ & $\begin{array}{c}V_{\min } \\
{[\mathrm{mag}]}\end{array}$ & $\begin{array}{c}P_{\text {puls }} \\
{[\mathrm{d}]}\end{array}$ & Ref. & $\begin{array}{l}P_{\mathrm{Bl}} \\
{[\mathrm{d}]}\end{array}$ & Ref. \\
\hline & & & & & & & & 504.03 & 25 \\
\hline ASAS174202-4633.7: & 174202.00 & -463342.0 & $\mathrm{RRc}$ & 10.74 & 11.11 & 0.3115788 & 25 & 1706.78 & 25 \\
\hline V829 Oph: & 174929.20 & 121354.5 & $\mathrm{RRab}$ & 13.5 & 15.0 & 0.56923 & 3 & 165 & 3 \\
\hline S Ara & 175910.73 & -492600.5 & $\mathrm{RRab}$ & 9.92 & 11.24 & 0.45186 & 17 & 49.37 & 25 \\
\hline AV Dra & 175944.21 & 515301.7 & RRab & 12.50 & 13.57 & 0.55560 & 3 & 96 & 27 \\
\hline ASAS180023-7026.5: & 180023.00 & -702630.0 & $\mathrm{RRc}$ & 12.08 & 12.47 & 0.3556146 & 25 & 1162.79 & 25 \\
\hline WW CrA & 180536.79 & -434957.4 & RRab & 11.66 & 12.48 & 0.55949 & 17 & 35.5 & 17 \\
\hline ASAS181215-5206.9 & 181215.00 & -520654.0 & RRab & 12.59 & 13.26 & 0.8375462 & 25 & 5.22 & 25 \\
\hline V442 Her: & 181258.32 & 420345.5 & $\mathrm{RRab}$ & 12.5 & 13.8 & 0.442084 & 3 & $\geq 700.00$ & 29 \\
\hline BD Dra & 181751.94 & 771749.2 & $\mathrm{RRab}$ & 12.05 & 13.01 & 0.58902 & 3 & 24.11 & 23 \\
\hline MW Lyr & 181953.82 & 315854.6 & $\mathrm{RRab}$ & 12.5 & 14.0 & 0.3976742 & 5 & 16.55 & 5 \\
\hline ASAS182913+2104.3 & 182913.00 & 210418.0 & RRab & 11.26 & 12.18 & 0.371117 & 3 & 23 & 27 \\
\hline KM Lyr: & 183029.76 & 401815.8 & RRab & 12.8 & 13.9 & 0.500193 & 3 & 30 & 26 \\
\hline KX Lyr: & 183315.22 & 401022.8 & RRab & 10.38 & 11.47 & 0.44090446 & 3 & $\ldots$ & 41 \\
\hline $\mathrm{BH}$ Pav & 183440.57 & -652703.0 & RRab & 11.5 & 13.1 & 0.4769536 & 25 & 173.7 & 25 \\
\hline AQ Lyr & 183451.04 & 263541.8 & RRab & 12.30 & 13.51 & 0.357134 & 8 & 64.9 & 8 \\
\hline CoRoT105288363 & 183930.86 & 072653.6 & RRab & 14.96 & 15.66 & 0.56744122 & 3 & 35.6 & 32 \\
\hline V413 CrA & 184757.62 & -374422.5 & $\mathrm{RRab}$ & 10.23 & 10.90 & 0.5893445 & 25 & 59.96 & 25 \\
\hline V349 Lyr: & 184924.27 & 424445.2 & $\mathrm{RRab}$ & 16.78 & 17.77 & 0.507074 & 31 & $\geq 127$ & 31 \\
\hline BD Her & 185032.19 & 163150.9 & $\mathrm{RRab}$ & 11.72 & 12.63 & 0.4739064 & 3 & $\sim 22$ & 8 \\
\hline V353 Lyr & 185201.78 & 451831.4 & RRab & 16.0 & 17.0 & 0.55682 & 31 & 60 & 31 \\
\hline V354 Lyr: & 185250.27 & 413349.4 & $\mathrm{RRab}$ & 15.0 & 16.0 & 0.56168 & 31 & $\geq 127$ & 31 \\
\hline V355 Lyr & 185325.83 & 430916.2 & $\mathrm{RRab}$ & 13.8 & 15.3 & 0.473697 & 31 & 31.4 & 31 \\
\hline ASAS185719-6321.4 & 185719.00 & -632124.0 & RRab & 12.28 & 13.24 & 0.41217 & 25 & 61.39 & 25 \\
\hline KIC11125706 & 190058.78 & 484441.6 & $\mathrm{RRab}$ & 11.83 & 12.26 & 0.61324 & 31 & 39.4 & 31 \\
\hline V360 Lyr & 190158.53 & 462645.7 & RRab & 15.5 & 16.5 & 0.55759 & 31 & 51.4 & 31 \\
\hline NR Lyr: & 190827.26 & 384846.0 & $\mathrm{RRab}$ & 12.22 & 12.98 & 0.6820264 & 3 & 27 & 27 \\
\hline V450 Lyr: & 190936.66 & 432150.0 & RRab & 14.3 & 16.7 & 0.50461 & 31 & $\sim 125$ & 31 \\
\hline V366 Lyr & 190940.65 & 461718.1 & RRab & 15.5 & 16.5 & 0.52702 & 31 & 65.6 & 31 \\
\hline V1104 Cyg & 191800.41 & 504517.5 & RRab & 14.5 & 15.5 & 0.43639 & 31 & 53.1 & 31 \\
\hline V1127 Aql & 192400.11 & 014148.9 & RRab & 14.8 & 16.0 & 0.355997 & 42 & 26.88 & 42 \\
\hline CoRoT100881648 & 192505.43 & 013923.8 & $\mathrm{RRab}$ & 14.94 & $\ldots$ & 0.60700 & 23 & 59.8 & 23 \\
\hline CoRoT101128793 & 192637.32 & 011334.9 & $\mathrm{RRab}$ & 15.93 & 16.53 & 0.4719296 & 3 & 18 & 33 \\
\hline ASAS192824-1852.4: & 192824.00 & -185224.0 & $\mathrm{RRc}$ & 12.65 & 13.10 & 0.3563567 & 25 & 1572.33 & 25 \\
\hline CoRoT101503544 & 192910.13 & 004346.9 & $\mathrm{RRab}$ & 14.52 & $\ldots$ & 0.60500 & 23 & 25.6 & 23 \\
\hline WY Dra & 193320.76 & 805542.9 & $\mathrm{RRab}$ & 12.08 & 13.64 & 0.588941 & 3 & 14.3 & 28 \\
\hline ASAS193538-7409.9: & 193538.00 & -740955.0 & RRc & 12.57 & 13.08 & 0.3499993 & 25 & 1608.49 & 25 \\
\hline V2178 Cyg: & 194006.99 & 385820.4 & RRab & 15.5 & 17.0 & 0.48680 & 31 & $\geq 200$ & 31 \\
\hline ASAS194502+2434.2 & 194502.00 & 243412.0 & $\mathrm{RRab}$ & 11.72 & 12.02 & 0.8458661 & 25 & 37.56 & 25 \\
\hline V808 Cyg: & 194539.07 & 393054.8 & $\mathrm{RRab}$ & 15.3 & 16.6 & 0.5478641 & 31 & $\sim 90$ & 31 \\
\hline FO Pav & 195142.20 & -624407.8 & $\mathrm{RRab}$ & 11.3 & 12.2 & 0.5514395 & 25 & 557.17 & 25 \\
\hline V783 Cyg & 195252.71 & 404735.4 & $\mathrm{RRab}$ & 14.2 & 15.5 & 0.6206994 & 31 & 27.7 & 31 \\
\hline ASAS195927-3400.1 & 195926.70 & -340003.5 & $\mathrm{RRab}$ & 11.88 & 12.65 & 0.37972 & 17 & 45.7 & 17 \\
\hline V759 Cyg & 200026.80 & 485937.8 & RRab & 12.1 & 13.8 & 0.360014 & 8 & 16 & 8 \\
\hline ASAS200431-5352.3 & 200431.40 & -535220.0 & $\mathrm{RRc}$ & 10.95 & 12.26 & 0.32402 & 25 & 10.82 & 25 \\
\hline KM Aql & 200556.27 & -083052.4 & RRab & 12.7 & 13.8 & 0.4381966 & 25 & 192.2 & 25 \\
\hline V2239 Sgr & 200909.67 & -414931.8 & $\mathrm{RRab}$ & 12.0 & 13.0 & 0.441943 & 25 & 45.39 & 25 \\
\hline V1645 Sgr: & 202044.47 & -410705.7 & $\mathrm{RRab}$ & 11.5 & 12.1 & 0.5529452 & 3 & 1331.74 & 25 \\
\hline GZ Del: & 202224.53 & 103407.3 & $\mathrm{RRab}$ & 15.4 & 16.7 & 0.33582841 & 3 & $\sim 36$ & 38 \\
\hline ASAS202746-2850.5: & 202745.70 & -285033.0 & RRab & 12.24 & 12.64 & 0.4084525 & 25 & 1674.48 & 25 \\
\hline
\end{tabular}


Table 1. continued.

\begin{tabular}{|c|c|c|c|c|c|c|c|c|c|}
\hline Star & RA & Dec & Type & $\begin{array}{c}V_{\max } \\
{[\mathrm{mag}]}\end{array}$ & $\begin{array}{c}V_{\min } \\
{[\mathrm{mag}]}\end{array}$ & $\begin{array}{c}P_{\text {puls }} \\
\text { [d] }\end{array}$ & Ref. & $\begin{array}{l}P_{\mathrm{Bl}} \\
\text { [d] }\end{array}$ & Ref. \\
\hline ASAS203145-2158.7 & 203145.00 & -215842.0 & $\mathrm{RRc}$ & 11.25 & 11.64 & 0.317152 & 25 & 792.83 & 25 \\
\hline ASAS203420-2508.9 & 203420.00 & -250854.0 & $\mathrm{RRab}$ & 11.58 & 12.48 & 0.5262389 & 25 & 666.44 & 25 \\
\hline ASAS203749-5735.5: & 203749.00 & -573530.0 & $\mathrm{RRc}$ & 12.38 & 12.66 & 0.4199162 & 25 & 1270.33 & 25 \\
\hline ASAS204440-2402.7 & 204439.90 & -240244.0 & $\mathrm{RRc}$ & 12.75 & 13.11 & 0.205333 & 25 & 6.64 & 25 \\
\hline FK Vul & 205231.00 & 222611.7 & RRab & 12.06 & 12.95 & 0.4340527 & 3 & 56 & 8 \\
\hline RV Cap & 210128.87 & -151346.1 & $\mathrm{RRab}$ & 10.22 & 11.57 & 0.4477465 & 25 & 231.66 & 25 \\
\hline ASAS210741-5844.2: & 210741.00 & -584412.0 & $\mathrm{RRc}$ & 13.33 & 13.78 & 0.3462376 & 25 & 1479.95 & 25 \\
\hline Z Mic: & 211622.71 & -301703.1 & $\mathrm{RRab}$ & 11.26 & 11.92 & 0.5869258 & 3 & $\ldots$ & 34 \\
\hline ASAS211839+0612.3: & 211839.40 & 061218.0 & $\mathrm{RRc}$ & 11.04 & 11.53 & 0.2914601 & 25 & 1176.75 & 25 \\
\hline ASAS212034+1837.2 & 212034.00 & 183712.0 & RRab & 11.50 & 12.26 & 0.5624065 & 25 & 81.3 & 25 \\
\hline DM Cyg & 212111.55 & 321128.7 & RRab & 10.93 & 11.99 & 0.419863 & 7 & 10.57 & 7 \\
\hline ASAS212331-3025.0: & 212331.00 & -302500.0 & $\mathrm{RRc}$ & 12.35 & 12.84 & 0.367442 & 25 & 1739.74 & 25 \\
\hline ASAS212433-5712.1 & 212433.24 & -571204.2 & $\mathrm{RRab}$ & 12.95 & 13.99 & 0.6051401 & 17 & 133.38 & 25 \\
\hline RY Oct & 213609.37 & -771813.5 & $\mathrm{RRab}$ & 11.46 & 12.46 & 0.563469 & 3 & 216.45 & 23 \\
\hline ASAS213826-3945.0: & 213826.00 & -394457.0 & $\mathrm{RRc}$ & 13.07 & 13.56 & 0.4107031 & 25 & 1540.12 & 25 \\
\hline ASAS214101+0109.6 & 214101.00 & 010936.0 & $\mathrm{RRab}$ & 12.43 & 13.0 & 0.6156709 & 25 & 522.58 & 25 \\
\hline RS Oct & 214716.91 & -873906.4 & RRab & 12.2 & 13.4 & 0.458038 & 3 & 244.2 & 25 \\
\hline RT Gru & 215158.44 & -455906.7 & $\mathrm{RRab}$ & 12.19 & 13.15 & 0.51216 & 17 & 87 & 17 \\
\hline SS Oct & 215335.38 & -824643.8 & RRab & 10.8 & 12.1 & 0.6218493 & 25 & 144.93 & 23 \\
\hline \multirow[t]{2}{*}{ BV Aqr: } & 220254.00 & -213132.1 & $\mathrm{RRc}$ & 10.72 & 11.24 & 0.363714 & 3 & 11.2 & 26 \\
\hline & & & & & & & & 1413.43 & 25 \\
\hline ASAS221556-2522.6 & 221555.70 & -252239.0 & $\mathrm{RRab}$ & 11.30 & 12.08 & 0.5467383 & 25 & 5.78 & 25 \\
\hline TY Gru: & 221639.42 & -395618.0 & $\mathrm{RRab}$ & 13.6 & 14.7 & 0.570076 & 3 & $\ldots$ & 34 \\
\hline GP Aqr: & 222539.14 & -075627.7 & $\mathrm{RRc}$ & 10.66 & 11.04 & 0.4052637 & 25 & 1618.65 & 25 \\
\hline AE Peg: & 222721.54 & 164816.7 & $\mathrm{RRab}$ & 11.83 & 13.15 & 0.4967235 & 3 & 23 & 27 \\
\hline ASAS223427-5635.4 & 223427.00 & -563524.0 & RRab & 13.07 & 13.45 & 0.61499 & 17 & 63 & 17 \\
\hline ASAS225131-3006.2: & 225131.00 & -300612.0 & $\mathrm{RRc}$ & 13.21 & 13.66 & 0.3384769 & 25 & 1681.8 & 25 \\
\hline ASAS225248-2442.2 & 225247.80 & -244212.0 & RRab & 12.78 & 13.97 & 0.5295565 & 25 & 181.2 & 25 \\
\hline BH Peg: & 225301.04 & 154716.6 & $\mathrm{RRab}$ & 9.99 & 10.79 & 0.640993 & 3 & 39.8 & 26 \\
\hline ASAS225323+0846.1 & 225323.20 & 084609.0 & $\mathrm{RRab}$ & 12.60 & 14.11 & 0.4930493 & 25 & 348.58 & 25 \\
\hline ASAS225518-2317.6: & 225518.00 & -231736.0 & $\mathrm{RRc}$ & 13.00 & 13.55 & 0.3935794 & 25 & 1557.88 & 25 \\
\hline BO Gru & 230658.64 & -435438.5 & $\mathrm{RRc}$ & 12.1 & 12.6 & 0.2811062 & 25 & 10.24 & 25 \\
\hline ASAS231209-1855.4: & 231209.00 & -185524.0 & $\mathrm{RRc}$ & 12.73 & 13.05 & 0.3079943 & 25 & 1349.89 & 25 \\
\hline GV And: & 231312.56 & 365404.0 & $\mathrm{RRab}$ & 13.07 & 14.03 & 0.528092 & 16 & $\sim 32$ & 16 \\
\hline ASAS232031-1447.9 & 232031.00 & -144754.0 & $\mathrm{RRab}$ & 12.46 & 13.05 & 0.6269552 & 25 & 54.52 & 25 \\
\hline ASAS233951-1644.4 & 233951.30 & -164425.0 & $\mathrm{RRc}$ & 12.16 & 12.68 & 0.3553741 & 25 & 875.96 & 25 \\
\hline DY And: & 235842.21 & 412919.4 & $\mathrm{RRab}$ & 12.94 & 14.15 & 0.6030897 & 3 & $\ldots$ & 35 \\
\hline
\end{tabular}

Table 2. Blazhko stars with multiple modulations.

\begin{tabular}{|c|c|c|c|c|c|c|c|c|c|c|}
\hline Star & RA & Dec & Type & $\begin{array}{c}V_{\max } \\
{[\mathrm{mag}]}\end{array}$ & $\begin{array}{c}V_{\min } \\
{[\mathrm{mag}]}\end{array}$ & $\begin{array}{c}P_{\text {puls }} \\
\text { [d] }\end{array}$ & Ref. & $\begin{array}{l}P_{\text {mod1 }} \\
{[\mathrm{d}]}\end{array}$ & $\begin{array}{c}P_{\text {mod2 }} \\
{[\mathrm{d}]}\end{array}$ & Ref. \\
\hline SU Col: ${ }^{a}$ & 050747.05 & -335154.5 & $\mathrm{RRab}$ & 11.32 & 13.33 & 0.4873552 & 25 & 65.41 & 88.98 & 25 \\
\hline UZ UMa & 081853.94 & +730547.8 & RRab & 13.10 & 15.00 & 0.4668413 & 6 & 26.7 & 143 & 8 \\
\hline LS Her & 160203.79 & +172850.4 & RRc & 11.04 & 11.53 & 0.230808 & 21 & 12.75 & 109 & 21 \\
\hline V872 Oph: & 175517.81 & +08 1342.9 & RRab & 14.70 & 15.80 & 0.45197319 & 3 & 13.5 & 51.13 & 40 \\
\hline RZ Lyr & 184337.88 & +324754.0 & RRab & 10.60 & 12.03 & 0.511230 & 11 & 121 & 30 & 11 \\
\hline V445 Lyr & 185825.59 & +413548.6 & $\mathrm{RRab}$ & 15.30 & 17.30 & 0.513075 & 31 & 53.2 & 143.3 & 31 \\
\hline XZ Cyg & 193229.31 & +562317.5 & RRab & 8.90 & 10.16 & 0.46659934 & 20 & 57.5 & 41.6 & 20 \\
\hline CZ Lac & 221930.76 & +512814.8 & RRab & 10.77 & 11.26 & 0.432174 & 30 & 14.6 & 18.6 & 30 \\
\hline
\end{tabular}

Notes. ${ }^{(a)}$ SU Col has one aditional modulation period $29.5 \mathrm{~d}$. 
Table 3. Stars with changing Blazhko period.

\begin{tabular}{lcccccccccc}
\hline \hline Star & RA & Dec & Type & $\begin{array}{c}V_{\max } \\
{[\mathrm{mag}]}\end{array}$ & $\begin{array}{c}V_{\min } \\
{[\mathrm{mag}]}\end{array}$ & $\begin{array}{c}P_{\text {puls }} \\
{[\mathrm{d}]}\end{array}$ & $\begin{array}{c}\text { Ref. } \\
P_{\text {BLmin }} \\
{[\mathrm{d}]}\end{array}$ & $\begin{array}{c}P_{\text {BLmax }} \\
{[\mathrm{d}]}\end{array}$ & Ref. \\
\hline AD UMa: & 092338.66 & +554633.2 & RRab & 15.0 & 16.3 & 0.548315 & 3 & 35 & 40 & 26 \\
RV UMa & 133318.09 & +535914.6 & RRab & 9.81 & 11.30 & 0.468060 & 3 & 89.9 & 90.63 & 12 \\
XZ Dra & 190942.61 & +645132.1 & RRab & 9.59 & 10.65 & 0.4764955 & 24 & 73 & 77 & 24 \\
RR Lyr & 192527.91 & +424703.7 & RRab & 7.06 & 8.12 & 0.566839 & 22 & 38.8 & 40.8 & 22 \\
\hline
\end{tabular}

References. (1) Phillips \& Gay (2004); (2) Jurcsik et al. (2006); (3) Samus et al. (2012) or Watson et al. (2006); (4) Jurcsik et al. (2005); (5) Jurcsik et al. (2008); (6) Sódor et al. (2006); (7) Jurcsik et al. (2009b); (8) Jurcsik et al. (2009a); (9) Sódor et al. (2012); (10) Szeidl et al. (2012); (11) Jurcsik et al. (2012); (12) Hurta et al. (2008); (13) Achtenberg \& Husar (2001); (14) Lee \& Schmidt (2001b); (15) Lee \& Schmidt (2001a); (16) Lee et al. (2002); (17) Sódor \& Wils (2005); (18) Kovács (2005); (19) Kolenberg et al. (2009); (20) La Cluyzé (2004); (21) Wils et al. (2008); (22) Kolenberg et al. (2006); (23) Le Borgne et al. (2012); (24) Jurcsik et al. (2002); (25) Szczygiel \& Fabrycky (2007); (26) Smith (1995); (27) Wils et al. (2006); (28) Chris et al. (1975); (29) Schmidt \& Lee (2000); (30) Sódor et al. (2011); (31) Benkö et al. (2010); (32) Chadid et al. (2011b); (33) Poretti et al. (2010); (34) For et al. (2011); (35) Cano \& Smith (2010); (36) Antipin et al. (2005); (37) Oppenheim \& Benson (1998); (38) Hacke \& Steiner-Sohn (1988); (39) Wunder (1990); (40) Hacke (1988); (41) Firmanyuk (1974); (42) Chadid et al. (2010); (43) Virnina et al. (2012); (44) Antipin et al. (2010).

\section{References}

Achterberg, H., \& Husar, D. 2001, IBVS, 5210

Antipin, S. V., Sokolovsky, K. V., \& Lebedev, A. A. 2005, IBVS, 5654

Antipin, S. V., Kazarovets, E. V., \& Pastukhova, E. N. 2010, PZM, 10, 33

Benkö, J. M., Kolenberg, K., Szabó, R., et al. 2010, MNRAS, 409, 1585

Blazhko S.N. 1907, AN, 175, 325

Buchler, J. R., \& Kolláth Z. 2011, ApJ, 731, 24

Cano, Z., \& Smith, R. C. 2010, Obs, 130, 11

Chadid M., Benkö J.M., Szabó, R. et al. 2011a, A\&A, 510, 39

Chadid, M., Perini, C., Bono, G., et al. 2011b, A\&A, 527, 146

Chis, D., Chris, G., \& Mihoc, I. 1975, IBVS, 960, 1

Dziembowski, W. A., \& Mizerski, T. 2004, AcA, 54, 364

Firmanyuk, B. N. 1974, ATsir, 828, 7

Fitch, W. S., Wisniewski, W. Z., \& Johnson, H. L. 1966, CoLPL, 5, 3

For, B-Q., Preston, G. W., \& Sneden, Ch. 2011, ApJS, 194, 38

Hacke, G. 1988, MitVS, 11, 102

Hacke, G., \& Steiner-Sohn, R. 1988, MitVS, 11, 123

Hurta, Zs., Jurcsik, J., Szeidl, B., \& Sódor, Á. 2008, AJ, 135, 957

Jerzykiewicz, M. 1995, ASPC, 78, 265

Jurcsik, J., Benkö, J. M., \& Szeidl, B. 2002, A\&A, 396, 539

Jurcsik, J., Sódor, Á., Váradi, M., et al. 2005, A\&A, 430, 1049

Jurcsik, J., Sódor, Á., Szeidl, B., et al. 2006, AJ, 132, 61

Jurcsik, J., Sódor, Á., Hurta, Zs., et al. 2008, MNRAS, 391, 164

Jurcsik, J., Sódor, Á., Szeidl, B., et al. 2009a, MNRAS, 400, 1006

Jurcsik, J., Hurta, Zs., Sódor, Á., et al. 2009b, MNRAS, 397, 350

Jurcsik, J., Sódor, Á., Hajdu, G., et al. 2012, MNRAS, 423, 993

Kolenberg, K. 2012, JAAVSO, 40, 481

Kolenberg, K., Smith, H. A., Gazeas, K. D., et al. 2006, A\&A, 459, 577

Kolengberg, K., Guggenberger, E., Medupe, T., et al. 2009, MNRAS, 396, 263
Kolenberg, K., Szabó, R., Kurtz, D. W., et al. 2010, ApJ, 713, L198

Kovács, G. 2005, A\&A, 438, 227

Kovács, G. 2009, AIPC, 1170, 261

LaCluyzé, A., Smith, H. A., Gill, E.-M., et al. 2004, AJ, 127, 1653

Le Borgne, J.-F., Klotz, A., Poretti, E., et al. 2012, AJ, 144, 39

Lee, K. M., \& Schmidt, E. G. 2001a, PASP, 113, 1140

Lee, K. M., \& Schmidt, E. G. 2001b, PASP, 113, 835

Lee, K. M., Schmidt, E. G., Langan, S. T., et al. 2002, PASP, 114, 546

Oppenheim, R., \& Benson, P. J. 1998, AAS, 192, 6718

Phillips, J., \& Gay, P. L. 2004, AAS, 205, 5404

Poretti, E., Paparó, M., Deleuil, M., et al. 2010, A\&A, 520, 108

Samus, N. N., Durlevich, O. V., Kazarovets, E. V., et al. 2012, CDS, The GCVS Catalog, B/gcvs (ver. 2012)

Schmidt, E. G., \& Lee, K. M. 2000, PASP, 112, 1262

Smith, H. A. 1995, RR Lyrae stars (Cam. Univ. Press)

Sódor, Á., \& Wils, P. 2005, IBVS, 5655

Sódor, Á., Vida, K., Jurcsik, J., et al. 2006, IBVS, 5705

Sódor, Á., Jurcsik, J., Szeidl, B., et al. 2011, MNRAS, 411, 1585

Sódor, Á., Hajdu, G., Jurcsik, J., et al. 2012, MNRAS, 427, 1517

Stothers, R. B. 2007, AJ, 652, 643

Szczygiel, D. M., \& Fabrycky, D. C. 2007, MNRAS, 377, 1263

Szeidl, B., Jurcsik, J., Sódor, Á., et al. 2012, MNRAS, 424, 3094

Virnina, N. A., Kocián, R., Hambálek, L. et al. 2012, OEJV, 146, 1

Watson, C. L., Henden, A. A., \& Price, A. 2006, in 25th Ann. Symp. Soc. Astron. Sci., 47

Wils, P., Lloyd, C., \& Bernhard, K. 2006, MNRAS, 368, 1757

Wils, P., Kleidis, S., \& Broens, E. 2008, MNRAS, 387, 783

Wunder, E. 1990, BAVRu, 39, 9 